Archives

13 | 1994

Enquêtes

\title{
Les cieux ouverts - les anges et leurs images dans le christianisme médiéval ( $\mathrm{XI}^{\mathrm{e}}$-XIII ${ }^{\mathrm{e}}$ siècles)
}

Étude d'anthropologie et d'iconographie religieuses

\section{Philippe Faure}

\section{(2) OpenEdition}

1 Journals

\section{Édition électronique}

URL : http://journals.openedition.org/ccrh/2699

DOI : $10.4000 /$ ccrh.2699

ISSN : $1760-7906$

Éditeur

Centre de recherches historiques - EHESS

\section{Édition imprimée}

Date de publication : 4 octobre 1994

ISSN : 0990-9141

\section{Référence électronique}

Philippe Faure, "Les cieux ouverts - les anges et leurs images dans le christianisme médiéval (XI siècles) », Les Cahiers du Centre de Recherches Historiques [En ligne], 13| 1994, mis en ligne le 27 février 2009, consulté le 03 mai 2019. URL : http://journals.openedition.org/ccrh/2699; ;OI : $10.4000 /$ ccrh.2699

Ce document a été généré automatiquement le 3 mai 2019.

Article L.111-1 du Code de la propriété intellectuelle. 


\section{Les cieux ouverts - les anges et leurs images dans le christianisme médiéval ( $\mathrm{XI}^{\mathrm{e}}-\mathrm{XIII}{ }^{\mathrm{e}}$ siècles)}

Étude d'anthropologie et d'iconographie religieuses

Philippe Faure

\section{NOTE DE L'AUTEUR}

Thèse de doctorat soutenue à l'EHESS en janvier 1994 sous la direction de Jean-Claude Schmitt

1 L'ange est une figure majeure de l'expérience religieuse au sein des religions du Livre, dans lesquelles angélologie et Révélation sont intimement liées. Historiens et philosophes des religions ont mis en évidence l'importance de l'ange comme figure médiatrice, entre le divin et l'humain. Étudiant le vaste domaine de l'islam iranien, Henry Corbin a montré combien l'angélologie fondait et mettait en mouvement la mystique, la philosophie spirituelle, la cosmologie et l'anthropologie. L'ange est conçu comme la forme spirituelle sous laquelle l'homme connaît Dieu et sous laquelle Dieu le connaît. Il apparaît comme manifestation de la Révélation et d'une connaissance initiatique, et comme pôle céleste de l'individualité humaine. L'identité entre angélophanie et théophanie se laisse saisir dans un espace-temps intermédiaire, un monde spécifique de l'image (âlam-al-mithâl), perçu par l'homme au moyen de l'imagination active. Henry Corbin a lancé des interrogations au sujet de la place de l'ange dans le christianisme occidental, du rapport entre l'affaiblissement ou la mutilation de celle-ci et la question de la sécularisation. Ce questionnement a surtout eu jusqu'ici un écho du côté des philosophes.

Or, la question de l'ange au sein du christianisme ne doit pas seulement relever de la philosophie, de la théologie ou de la spiritualité, mais d'une approche historique et anthropologique. Chacun peut constater l'épanouissement de la figure angélique, dans les multiples expressions de la civilisation médiévale, et son caractère résiduel dans le 
christianisme contemporain. Il s'agit donc de faire de l'ange un objet d'histoire, de poser les problèmes de la fonction de la croyance et de son importance. On s'est employé à analyser le fonctionnement de la thématique angélique et ses rapports avec la doctrine ou avec les orientations de la piété. On a cependant laissé de côté, pour l'essentiel, la question des conceptions hérétiques ou païennes des êtres invisibles.

Le fait que le christianisme soit fondé sur le mystère de l'Incarnation ne saurait dispenser d'une enquête sur la place de l'ange en son sein. Il existe une tradition angélologique chrétienne, que des études pionnières (J. Tunnel, G. Tavard) ont mises en évidence, indiquant déjà que l'enseignement doctrinal avait connu des variations non négligeables. Partant de l'intuition que la permanence et l'omniprésence de la figure angélique masquait le fait que les anges avaient une histoire dans le cadre du christianisme occidental, on a estimé qu'une étude consacrée aux rapports entre l'ange et l'homme durant la période médiévale serait riche d'enseignements: elle pouvait contribuer à délimiter le champ d'extension, les modalités d'expression et les répercussions anthropologiques d'une thématique spirituelle jusqu'alors négligée. Elle pouvait nous renseigner sur son rôle, sur ses évolutions, sur les mutations culturelles à l'œuvre, dans la mesure où elle subit des inflexions ou des tendances révélatrices de mutations plus larges et décisives, du $\mathrm{x}^{\mathrm{e}}$ au XIII ${ }^{\mathrm{e}}$ siècle.

Deuxièmement, une telle enquête pouvait apporter une contribution à la compréhension de l'image religieuse. L'ange est assurément messager, comme le souligne l'étymologie (l' angelos grec traduisant le maleak hébreu), mais pour être messager, il faut être. Premier miroir de la divinité, l'ange est par nature lié à la question de l'image. L'ange relève des trois mondes de l'image : image de la divinité, image visionnaire et subtile, image figurée et sensible. L'étude des représentations textuelles et des images figurées donne une épaisseur à la notion de représentation, permet d'approcher les questions de la corporéité ou du rapport entre la parole et l'image de l'ange. En employant les notions d'angélophanies et de théophanies, maniées par l'histoire des religions, on a eu le souci d'apprécier l'intensité de la manifestation angélique et sa signification, dans les textes et dans les images. L'enjeu est de parvenir, à partir de l'analyse des modes de figuration, non seulement à la mise en lumière d'un message, d'une sensibilité, d'un mode de pensée, mais à évaluer leur impact et à les saisir comme indice d'une mentalité ou d'une mutation culturelle.

5 Du côté de l'iconographie, quelques travaux fournissaient des analyses intéressantes, mais il leur manquait un élargissement d'investigation et une mise en perspective historique. En outre, le recours aux sources iconographiques est apparu essentiel pour saisir les tenants et les aboutissants de la représentation de l'ange. Celui-ci n'est pas seulement un élément du système religieux, mais une image où prend corps un champ de l'imaginaire, ou, pour reprendre l'expression forgée par Henry Corbin, une part du « monde imaginal » perçu par l'imagination active. Il ne s'agit pas seulement d'analyser la cohérence et la richesse symbolique des représentations, mais de repérer les transformations qui affectent les mises en forme de cet imaginaire, et notamment la diversité et l'évolution des modèles.

6 Enfin, en contribuant à une histoire des conceptions et représentations des esprits célestes et de leurs relations avec les hommes, on a souhaité nourrir une histoire comparée des religions, soucieuse de procéder à une évaluation de l'angélologie au sein des trois rameaux du monothéisme sémitique. 
7 La problématique choisie posait un certain nombre d'exigences : il convenait de prendre le sujet globalement, c'est-à-dire de tenter d'évaluer la thématique angélique dans son ensemble, sous peine de ne pouvoir tirer des conclusions relevant de l'anthropologie religieuse. Il fallait par conséquent se situer spatialement au plan de l'Occident latin, et couvrir une période suffisamment large pour espérer prendre la mesure des caractéristiques et des mutations de la présence angélique. Mais, sous peine de se disperser, de juxtaposer les études particulières, de ne pouvoir maîtriser une documentation surabondante, il fallait aussi se limiter.

8 On a donc opéré des choix; choix des sources tout d'abord: la perspective d'une étude centrée sur le thème du rapport entre l'ange et l'homme et sur le thème de l'image, dans ses différentes formes, m'a amené à solliciter aussi bien des images que des textes. La problématique a conduit à privilégier les récits de visions ou d'apparitions, les vies de saints, recueils de miracles et exempla, les traités mystiques et les encyclopédies. Cette variété permettait de distinguer des niveaux d'utilisation de la thématique angélique et de prendre la mesure d'une réalité historique complexe. En ce qui concerne les images, on a puisé essentiellement dans l'illustration des manuscrits enluminés, Bibles et ouvrages liturgiques, et de quelques vies de saints où les apparitions angéliques occupent une place remarquable. Cela a permis de juger des fonctions de l'ange et de son mode de présence, d'évaluer la signification de celle-ci et de mieux cerner le rapport entre l'image et les textes qui s'y rattachent, directement ou non. Il n'était pas réaliste d'embrasser la totalité de l'iconographie de l'ange ; aussi a-t-on choisi de suivre des thèmes connus et diffusés, qui permettaient d'envisager les principaux aspects de la thématique angélique, de voir en quoi elle a pu former un système de représentation et en quoi elle a subi des évolutions. On a examiné quelques programmes iconographiques complets, de manière à approcher les modes d'utilisation et le fonctionnement interne de la présence angélique. Mais on s'est également penché sur des thèmes à diffusion plus restreinte (les neuf choeurs, Lucifer) dont le traitement iconographique est apparu d'une grande portée sur le plan de l'anthropologie religieuse.

9 La place accordée aux images correspond au souci de leur donner toute leur importance dans une histoire des représentations, mais aussi de montrer leur spécificité. Les phénomènes iconographiques sont historiques; ils expriment une conception et une vision propres à un temps et à une société, mais selon des règles et des modalités particulières. On s'est donc efforcé de comprendre le langage des images, leurs structures et leurs modes de fonctionnement, sans oublier leur contexte.

Spatialement, on a privilégié l'Europe du nord-ouest (France, îles britanniques et pays germaniques), et chronologiquement, le Moyen Age central. Cette double orientation s'explique par le souci de constituer des séries documentaires et de saisir les formes d'expression et les évolutions de la thématique angélique dans une période marquée, chacun le sait, par une inventivité et des mutations remarquables. Il était en particulier important de confronter la thématique angélique à l'affirmation d'un christocentrisme de plus en plus net après l'an mille et de la dévotion à l'humanité du Christ. Mais on a ouvert l'enquête sur les XIV ${ }^{e}$ et $\mathrm{XV}^{\mathrm{e}}$ siècles, qui voient s'enclencher de profondes mutations de la thématique angélique.

11 Dans le cadre de cette présentation, on ne peut aborder toutes les questions posées par les divers types de documents, ni traduire la richesse thématique des textes et des images. On s'attachera cependant à dessiner les contours de la thématique angélique, telle qu'elle 
se laisse saisir dans ses expressions multiples, et de l'évolution des représentations, tel qu'on peut la déceler à partir du XII ${ }^{\mathrm{e}}$ siècle.

\section{La tradition angélologique occidentale}

12 L'an mille est le terminus a quo de cette étude. Ce point de départ, commode pour espérer saisir les mutations du Moyen Age central, demande une mise au point préliminaire. La tradition angélologique est en effet fixée dès le début du Haut Moyen Age, puis développée au $\mathrm{IX}^{\mathrm{e}}$ siècle. En Occident, Saint-Augustin a jeté les bases de l'angélologie médiévale. Il lègue notamment une interprétation angélologique du thème de la lumière, de l'origine du mal et de la connaissance de Dieu. Il énonce les principales fonctions des êtres célestes, tournés à la fois vers Dieu, qu'ils glorifient, et vers le monde, qu'ils soustendent et dans lequel ils interviennent. L'évêque d'Hippone met aussi en valeur la correspondance profonde qui unit l'ange et l'homme: les deux créatures sont intelligentes et créées à l'image de Dieu, mais l'une est spirituelle, l'autre corporelle.

Deuxième pilier de la tradition angélologique occidentale, Grégoire le Grand reste fidèle à l'enseignement d'Augustin, qu'il développe sur certains points. C'est lui qui répand l'idée selon laquelle l'homme est appelé à combler au ciel les places abandonnées par les anges déchus. C'est encore lui qui énumère, dans plusieurs listes, les neuf choeurs célestes, dont il a pris connaissance lors d'un voyage à Constantinople. Mais il n'en tire pas un enseignement mystique. Pour cela, il faut attendre le $\mathrm{IX}^{\mathrm{e}}$ siècle et l'arrivée en Occident des œuvres de Denys, le pseudo-Aréopagite, traduites et commentées par Jean Scot Erigène. Si cette irruption n'a pas d'effets immédiats, elle n'en est pas moins un fait essentiel dans la longue durée. La notion de "hiérarchie céleste» donnera lieu à de multiples développements, aussi bien politiques que mystiques et intellectuels, d'une complexité croissante.

Le Haut Moyen Age a vu s'enraciner en Occident le culte des archanges, et singulièrement de Saint Michel, à partir de l'Italie (Monte Gargano) et des terres celtiques (Mont Saint Michel). C'est en tant que puissance divine, gardienne du peuple chrétien et luttant contre le démon, que l'archange est ainsi valorisé. De nombreuses chapelles hautes, des peintures murales et des sculptures en témoignent abondamment. Ce culte, étendu en 813 à tout l'empire carolingien, est l'élément le plus visible d'une dévotion aux anges que développe le monachisme bénédictin. Il dénote aussi la volonté de limiter et contrôler le culte des êtres invisibles, car il a permis à l'Église de canaliser des tendances polythéistes et de christianiser des lieux autrefois consacrés aux dieux gallo-romains ou à des génies locaux. Comme l'a montré K. Heitz, les tourelles angéliques des monastères, d'abord indépendantes des églises, ont été progressivement intégrées aux édifices. Au VIII ${ }^{\mathrm{e}}$ siècle, l'hérésie d'Adalbert, qui répandait une prière contenant des noms d'anges tout à fait fantaisistes, a fourni à la papauté l'occasion de limiter officiellement la dévotion aux trois archanges expressément nommés dans les Écritures.

Cette mise en ordre a concerné le clergé lui-même, au sein duquel les noms et les figures d'anges, même non canoniques, étaient utilisés comme puissances protectrices: le tombeau de l'abbé Mellebaude, dans l'Hypogée des Dunes, à Poitiers, en est la preuve. La spiritualité bénédictine va peu à peu diffuser un mode de relation plus fraternel entre les anges et les hommes, qui permet d'esquiver le recours aux noms. Ce qui compte, en effet, 
n'est pas la puissance active, mais la liaison fréquente, dans la liturgie, entre les créatures, et leur réunion eschatologique.

En ce qui concerne l'iconographie, il faut d'abord souligner qu'à aucun moment la représentation des anges n'est mise en cause, en dépit de la querelle des images, et qu'elle peut même se prévaloir de la légitimité que lui accorde le concile de Nicée II (787). Ses canons, reçus en Occident mais vivement contestés sous Charlemagne, puis peu à peu tombés dans l'oubli, ne semblent pas avoir influencé les représentations de l'ange. Celles$\mathrm{ci}$, à la fin $\mathrm{du} \mathrm{x}^{\mathrm{e}}$ siècle, sont essentiellement issues de modèles orientaux, mis au point dans l'empire byzantin, et qui, par l'Italie, ont gagné la majeure partie de l'Occident, notamment les pays germaniques. Les attributs angéliques, les vêtements, les insignes que l'on découvre dans la miniature sont empruntés à la peinture orientale. Mais les mises en scène sont bien souvent retravaillées, et les traditions occidentales, notamment insulaires, sont loin d'être négligeables. La codification de l'angélologie et d'une spiritualité monastique où l'ange joue un rôle essentiel conduisent à la formation d'un système angélophanique dont il convient d'analyser les rouages.

\section{L'affirmation d'un système angélophanique.}

L'ange est conçu comme un être dont la vocation est de se manifester aux hommes et d'entretenir des relations avec eux. Aussi les textes comme les images donnent toute son ampleur à un espace-temps intermédiaire, qui permet de manifester la divinité et de diviniser le monde humain.

Le plus frappant est la fonction «polaire » du monde angélique, telle qu'elle s'exprime dans la dimension "mythique» d'une histoire céleste fondatrice, qui commande le rapport des hommes au ciel spirituel et au monde terrestre, et qui fonde la démonologie. L'iconographie de l'histoire de Lucifer ou celle des neuf choeurs célestes montre qu'il ne s'agit pas de croyances accessoires, mais bien d'une histoire dont l'enjeu est le rattachement de l'homme au ciel. L'espace-temps visionnaire concerne la divinité ellemême, dans la mesure où l'iconographie utilise l'ange comme figure de Dieu. Le sens théophanique de l'être céleste traduit l'importance qui est la sienne comme forme spirituelle.

Dans le contexte d'un christianisme centré sur l'eschatologie et sur la vision anticipée de la Jérusalem céleste, la figure angélique prenait une dimension exceptionnelle. Le plus éclatant témoignage en est, au XII ${ }^{\mathrm{e}}$ siècle, la fresque de la tribune du transept nord de Notre-Dame du Puy en Velay. L'archange Saint-Michel, qui piétine le dragon, atteint cinq mètres de haut : c'est le personnage le plus grand de la peinture murale française. L'être céleste est une figure théophanique, qui exprime l'action divine. Les anges qui ornent les coupoles des églises sont les habitants d'une cité céleste que les hommes sont appelés à rejoindre; et la liturgie est le temps privilégié qui permet l'union des deux types de créatures, céleste et terrestre. La symbolique architecturale fait de l'édifice cultuel l'image parfois exemplaire, comme à Saint-Benoît-sur-Loire, de la Jérusalem céleste, dont l'Apocalypse révèle la forme, les proportions et le nombre des portes.

20 Le thème des quatre archanges ou des huit anges, que l'on découvre dans la chapelle palatine de Palerme ou à Ratisbonne, constitue une autre expression de la souveraineté céleste et du royaume de Dieu, mise au service d'idéologies royales. 
21 La conviction que l'homme est destiné à remplacer au ciel les anges déchus fonde une véritable "anthropologie angélique ", pour reprendre l'expression forgée naguère par Robert Bultot. Cette polarité spirituelle donne un sens supérieur à l'être humain et commande une conception des rapports de l'homme avec le monde. Jusqu'au XII siècle, l'idéal monastique, installé dans une position dominante, est considéré comme la traduction terrestre la plus parfaite de la vie angélique. Par la virginité, la contemplation et la célébration de la gloire de Dieu, le moine est assimilé à l'ange. La psalmodie communautaire reflète ici-bas la vie céleste ; elle est comme une ouverture du ciel sur la terre, qui réunit les anges et les hommes. Le chorus est cette actualisation liturgique du royaume de Dieu ; il anticipe la réunion promise à la fin des temps. Par la mise en œuvre de codes symboliques, l'iconographie met en valeur cette dimension. L'illustration du De Laudibus sanctae crucis de Raban Maur souligne que les neuf choeurs angéliques et Adam se trouvent réunis dans une même louange, Alleluia-Amen, exprimée dans la forme de la croix. La conviction que la vie monastique est l'expression la plus pure de la polarité angélique de l'homme s'accompagne d'un discours négatif à l'égard du monde et de tous les comportements qui relèvent de la nature, comme en témoigne l'œuvre de Pierre Damien au début du xi ${ }^{\mathrm{e}}$ siècle.

L'ange est conçu non seulement comme modèle céleste, mais comme compagnon spirituel et initiateur. Issue de la tradition du désert, cette conception est mise en valeur dans les récits de visions. L'ange est à la fois un idéal spirituel et l'agent du dévoilement des mystères célestes, donc de l'acquisition d'une connaissance illuminatrice. La manifestation angélique est une descente du ciel sur la terre qui sanctifie l'homme et le temps. Cette opération spirituelle concerne l'espace naturel, puis l'espace ecclésiastique.

La visite et la vision des anges apparaissent comme le signe de la sainteté du moine et de sa ressemblance aux habitants du ciel, qui le reconnaissent pour frère. Dans les vitae anglo-irlandaises du Haut Moyen Age, les anges sont autant sur terre que dans le ciel et toujours prêts à se manifester aux hommes de Dieu.

Dans l'ensemble des sources consultées, il faut souligner le poids des modèles vétérotestamentaires. La mise en scène des rapports entre l'ange et l'homme s'enracine alors dans les épisodes bien connus, tels que l'hospitalité d'Abraham, la lutte de Jacob, la vision de l'échelle, les annonciations aux prophètes. Ce n'est que peu à peu que les données du Nouveau Testament sont prises en compte et conduisent notamment à des formules iconographiques nouvelles. Le meilleur exemple en est sans doute la scène de l'Annonciation, qui ne se distingue pas, avant le $\mathrm{xI}^{\mathrm{e}}$ siècle, des autres scènes d'annonce angélique, mais qui acquiert progressivement une spécificité croissante, que l'on doit mettre en rapport avec l'orientation nouvelle de la piété, centrée sur le mystère de l'Incarnation.

\section{Modifications graduelles du système angélophanique}

$25 \mathrm{Au} \mathrm{XI}^{\mathrm{e}}$ siècle, la réflexion théologique sur les anges s'est approfondie grâce à SaintAnselme, qui médite sur la chute du diable et sur le destin spirituel de l'homme. La correspondance suivie de l'état angélique et de l'état humain sous-tend toute son œuvre, mais il rejette toute relation de causalité entre la chute des anges et la création d'Adam. L'homme doit compléter au ciel le nombre des anges, mais Dieu l'a créé pour lui-même, et non seulement pour remédier au drame céleste. La montée en puissance de l'hérésie met 
en valeur le rôle de la thématique angélique dans les conceptions métaphysiques hétérodoxes et le danger de spéculations dans ce domaine. $\mathrm{C}^{\prime}$ est cependant durant les $\mathrm{XI}^{\mathrm{e}}$ et $\mathrm{XII}^{\mathrm{e}}$ siècles que la figure angélique semble avoir trouvé durablement sa place dans l'expérience religieuse et dans les expressions artistiques de la foi Les images des anges se multiplient, mais à l'intérieur de scènes qui mettent en jeu Dieu et les hommes. Entre les deux, l'ange a trouvé sa place et ses fonctions.

Celles-ci apparaissent fondamentales : les anges sont inséparables de la manifestation de Dieu en gloire ; ils permettent de représenter le drame de l'origine du mal et de la fin des temps; ils sont les messagers de la volonté divine, les révélateurs de secrets célestes, les guides et les compagnons de l'homme, ici-bas et dans l'Au-delà. Leur rôle n'est pas seulement de mettre en mouvement les images et de les rendre narratives, par un code symbolique approprié, mais de dévoiler temporairement un espace et un temps autres que l'espace-temps terrestre.

Les visions d'Hildegarde de Bingen sont à bien des égards angélomorphiques, même si la figure angélique y est remodelée par une imagination symbolique puissamment originale. D'autre part, Honorius rejette, comme Saint-Anselme, la relation de causalité entre la chute des anges et la création d'Adam, et il accorde davantage d'intérêt à la nature Ces éléments nouveaux annoncent une modification du système issu de l'anthropologie angélique.

Celle-ci évolue dans le courant du XII ${ }^{\mathrm{e}}$ siècle, qui voit l'émergence d'une relation plus personnelle entre l'homme et l'ange, en particulier dans la notion d'ange gardien, à la fois protecteur et ami. A côté du protecteur collectif qu'est Saint-Michel prend place un gardien individuel. Saint-Bernard accorde une large place aux anges dans l'ascension mystique ; ils préparent l'âme à la vision de Dieu et lui présentent des images des réalités célestes. L'abbé de Clairvaux évoque aussi les anges gardiens, mais c'est dans l'Elucidarium d'Honorius augustodunensis que l'on trouve le premier exposé précis à leur propos. Les prières à l'ange gardien se multiplient, les récits de visions font état d'un contact plus personnalisé et plus affectif avec l'être céleste. Elisabeth de Schonau évoque à plusieurs reprises « son ange » (meus angelus); l'iconographie commence à présenter des exemples qui traduisent la même idée, notamment dans le cas de la relation entre Tobie et l'archange Raphaël.

29 Cette émergence est sans doute à rapprocher d'une certaine " découverte de l'individu » au XII ${ }^{\mathrm{e}}$ siècle. Mais il importe de souligner que le protecteur et guide personnel n'a qu'un rôle limité et surtout qu'il est relié à un monde angélique hiérarchisé. Jusqu'au milieu du $\mathrm{XII}^{\mathrm{e}}$ siècle, cette hiérarchie est encore envisagée comme une superposition graduée d'êtres célestes.

Ces évolutions semblent également liées à l'insistance de la prédication sur le jugement personnel après la mort et à la transformation de l'Au-delà. L'affirmation du Purgatoire à la fin $\mathrm{du} \mathrm{XII}^{\mathrm{e}}$ siècle valorise nettement le rôle de l'ange gardien, dont l'activité s'étend aux âmes en souffrance.

31 L'iconographie subit aussi l'influence de l'essor de la dévotion mariale et de la prédication. La dimension visionnaire de la manifestation angélique s'estompe, avec l'aide des tendances rationalisantes de la culture religieuse. Dans les traités de morale, le chérubin devient une figure allégorique, dont les ailes deviennent les supports des noms des vertus chrétiennes. 


\section{Tensions, approfondissements et mutations au XIII siècle}

De nouvelles évolutions marquent le système angélologique occidental à partir de la fin du XII siècle, sous l'effet de plusieurs facteurs.

L'introduction de l'œuvre d'Avicenne met les théologiens latins en présence d'une angélologie musulmane, à laquelle s'attaque Guillaume d'Auvergne dans son De Universo. Aristote et Averroës introduisent la notion d'intelligence séparée, qui permet de reconsidérer la nature angélique. Le développement de l'hérésie cathare, dans laquelle l'angélologie tient une place essentielle, conduit l'Église à formuler pour la première fois, au concile de Latran IV (1215), sa doctrine sur la création des êtres invisibles. Il s'agit d'affirmer l'unité foncière de l'univers créé et le libre arbitre angélique. Les commentaires de Joachim de Flore, puis des Spirituels franciscains, sur l'Apocalypse de Jean et sur la figure de l'Ange du sixième sceau $($ Ap. 7,2$)$ nourrissent un imaginaire mystique et une spéculation hardie sur le sens de l'histoire. Saint François est ainsi identifié à cet ange, qui ouvre l'âge de l'Esprit.

Traduit et commenté par Jean Scot Erigène au milieu du IX $\mathrm{I}^{\mathrm{e}}$ siècle, mais sans postérité notable, La Hiérarchie céleste du Pseudo-Denys l'Aréopagite fait l'objet de traductions et d'exégèses nouvelles Hugues de saint Victor dès 1137, puis, au XIII ${ }^{\mathrm{e}}$ siècle, Albert le grand, Robert Grosseteste, Bonaventure, commentent l'ouvrage et l'utilisent largement dans leurs propres œuvres. Thomas d'Aquin lui-même, dont on ne souligne trop souvent que l'aristotélisme, cite Denys plus encore que le Stagirite.

5 La maturation intellectuelle induite par ces apports multiples conduit à une nouvelle formulation de l'angélologie chrétienne, non seulement dans les grandes sommes de théologie, mais aussi dans les ouvrages encyclopédiques, dont le Speculum naturale de Vincent de Beauvais est, à la fin du XIII ${ }^{\mathrm{e}}$ siècle, le meilleur exemple. Ce sont les deux idées de lumière et de hiérarchie qui semblent avoir le plus bénéficié du travail de réflexion entrepris à partir du corpus dionysien. Ces deux thèmes se conjuguent dans la figure de l'ange d'une manière nouvelle au XIII ${ }^{\mathrm{e}}$ siècle. L'iconographie des neuf choeurs célestes montre que la hiérarchie angélique est comprise dans un sens de participation mystique à la vie divine et d'irradiation graduelle de la lumière spirituelle. Le vitrail angélique du transept sud de Chartres, la coupole du baptistère de Florence, en témoignent avec éclat, grâce à une savante mise en scène qui suggère l'image de la spirale.

La dimension "mythique» s'estompe au profit d'un souci de traduire la fécondation céleste de l'histoire par l'irruption soudaine de l'ange. Celui-ci est le déclencheur d'une mutation temporelle qui dénote une nouvelle attention à l'histoire et l'attente d'un temps nouveau, de nature spirituelle.

Mais la présence angélique tend à s'articuler étroitement à la dévotion à l'humanité du Christ souffrant L'humanisation de l'ange est le corollaire de celle-ci; l'anthropologie angélique se transforme dans son contenu et ses modes d'expression. La méditation du sacrifice du Christ semble permettre la manifestation des anges supérieurs : les séraphins, formes ultimes des états spirituels et de l'amour de Dieu.

L'accroissement quantitatif de la présence angélique dans les images traduit un désir de voir les habitants du ciel accompagner les hommes sur la terre. L'essor de la dévotion aux anges gardiens et, à l'autre extrémité de l'échelle céleste, la manifestation visionnaire des

Les Cahiers du Centre de Recherches Historiques, 13 | 1994 
séraphins en sont deux expressions essentielles. Ce compagnonnage vertical ou supra humain vient compléter le compagnonnage des fidèles tel qu'il se manifeste dans l'apparition des confréries; il est aussi en rapport avec l'ampleur que prend la prédication. Les exempla diffusent un usage raisonné de l'ange, surtout considéré comme un serviteur et un conseiller qui exhorte à la pratique sacramentelle. Il apparaît comme un moyen de perfectionnement personnel et de surveillance céleste. La pédagogie angélique s'exprime dans la mise en jeu des images : la Bible moralisée met clairement en rapport anges et prédicateurs.

Les apports culturels nouveaux, la pression de l'hérésie, l'influence dionysienne, l'apparition des Ordres Mendiants, le rôle accru des laïcs éclairent les changements constatés dans le système angélophanique.

La lumière rejoint la piété mariale en plein essor, pour faire de l'ange, serviteur de la Vierge, un être plus féminin, aux traits plus doux, au corps plus fin, plus léger, à la longue chevelure. Le diptyque de Wilton (1390) porte à son paroxysme cette image féminine de l'ange. Les visions de Mechtilde de Hackeborn montrent que les anges baignent dans la lumière céleste et sont inséparables de la manifestation hiérarchique de la gloire divine. L'expérience de François d'Assise, souvent réduite à la réception des stigmates de la Passion du Christ, apparaît d'abord comme la réalisation de l'homme hiérarchique. Les voies de la mystique rejoignent ainsi le discours purement doctrinal des scolastiques. Mais la luminosité et la féminité relative de la figure angélique s'accompagnent de l'expression des sentiments humains. Il ne faudrait pas seulement évoquer «l'ange au sourire » de la cathédrale de Reims, mais aussi « les anges aux pleurs» des crucifixions peintes par Duccio, Cimabue ou Giotto.

41 Les anges agissent comme les révélateurs de la tonalité du sentiment religieux de la société. L'apparition de l'ange gardien sous la forme de l'enfant, chez Mechtilde ou chez Gertrude d'Helfta, semble traduire d'une autre manière la valorisation de la féminité, de la douceur et de l'amour. Le thème de l'exaltation de la Vierge «au-delà des chœurs angéliques" fait d'elle la mère de toutes les créatures célestes et pas seulement des hommes. Cette reformulation de la parenté des anges et des hommes par la piété mariale semble avoir préparé « l'infantilisation » de l'image de l'ange à la fin du Moyen Age.

Le recul de l'idéal de la réalisation monastique, où l'ange apparaissait comme le modèle spirituel à imiter, l'insistance nouvelle sur la nature humaine du Christ et les souffrances de la Passion, la reconnaissance plus grande de la nature ont-ils conduit à un abandon de l'anthropologie angélique? Il semble bien que l'on assiste plutôt à un élargissement de ses modalités. L'imitation du Christ devient le pivot de la dévotion, à partir duquel doit se redéfinir l'imitation des anges. L'itinéraire de Saint-François est exemplaire, en ce sens que coexistent en lui l'imitation des anges et l'imitation du Christ, réalisées dans son corps l'une et l'autre, sans contradiction; la stigmatisation vient parachever une réalisation de l'homme angélique. Mais on a en général plutôt l'impression d'une distinction croissante entre les deux formes de piété.

La montée en puissance des élites laïques, l'essor d'une piété plus individuelle, la miniaturisation des supports de dévotion conduisent au XIV siècle à une modification importante du système angélologique et du langage des images. Le fond et la forme se conjuguent pour produire un affaiblissement de la figure angélique. Son caractère théophanique tend à disparaître, au profit de l'expression des sentiments humains et des 
attitudes religieuses du fidèle. L'humanité des anges suit l'humanité du Christ et de la Vierge.

\section{Le triomphe des anges gardiens} plus humaine la figure angélique. fête de l'ange du Portugal (1504). l'angélologie médiévale à la piété moderne. bienfaisants.

Les images de dévotion destinées aux laïcs de haut rang sont caractérisées par l'emploi d'un code de signes qui provoquent l'amoindrissement de la figure angélique dans sa fonction de médiateur et de messager de Dieu. La transmission, fonction pourtant essentielle de l'ange, passe par d'autres canaux, et par Dieu le Père en personne. L'être céleste ne semble garder son importance que comme escorte lumineuse de Dieu, dans les hauteurs célestes, et comme protecteur, individuel ou collectif.

La fin $\mathrm{du} \mathrm{XIV}^{\mathrm{e}}$ siècle est à cet égard un moment décisif. C'est en Aragon que semble prendre son essor la nouvelle dévotion aux anges gardiens, en partie sous l'influence du franciscain Francesco Eiximenis et du dominicain saint Vincent Ferrier. Il s'agit d'abord d'un culte rendu aux anges des cités, comme à Valence, en 1390. Selon Jean Delumeau, le besoin de sécurité face aux calamités du temps, et à la peste en particulier, est à l'origine de la promotion de cette vénération. Mais il faut sans doute y voir aussi une forme de reconnaissance de la ville comme image de la Jérusalem céleste. Réunie derrière ses murs, sous la protection de son ange, la société urbaine reflète l'assemblée des habitants de la cité sainte. Le fragile bonheur des citadins a pu susciter le besoin de rendre plus proche et

L'ange est, avec la Vierge Marie et les saints l'une des figures essentielles capables de répondre à la montée d'une piété qui se manifeste dans l'appartenance à une nation, à un groupe social, à une confrérie. Dans la péninsule ibérique, la fin de la période médiévale coïncide avec la généralisation du culte aux anges des cités et avec la proclamation de la

La méditation de la notion d'ange gardien individuel est aussi relancée à la fin du XIV siècle, par les sermons de Ludolphe le Saxon, de Vincent Ferrier, de Jean Gerson, et elle aboutit à la reconnaissance de la première fête solennelle des anges gardiens par une bulle pontificale de 1518, à la demande de l'évêque de Rodez, François d'Estaing. Ainsi, la dévotion aux anges gardiens, individuels ou collectifs, apparait comme le legs essentiel de

Chez Gerson, cette dévotion est encore reliée au souci de l'ascension mystique, conformément à la perspective dionysienne, mais il semble que l'ange apparaisse le plus souvent comme une réponse au besoin de protection individuelle. Deux aspects se dégagent de cette figure : le gardien-combattant et le guide de l'âme. Le premier trait, plus précoce, s'enracine dans la personnalité guerrière de Saint-Michel et prend place dans une vision du monde comme théâtre d'un combat sans merci entre les anges et les démons. L'omniprésence angélique semble faire écho à une omniprésence du diable. Il est difficile de ne pas déceler, derrière "l'explosion démographique des anges", dans les enluminures et dans les marges des livres d'heures, supports de la piété des élites laïques, une réponse aux peurs et à l'angoisse du salut. L'univers angélique fournissait un inépuisable réservoir d'intercesseurs et la vision rassurante d'un univers peuplé d'esprits

Issu de la figure biblique de l'archange Raphaël, promu patron des voyageurs au $\mathrm{xv}^{\mathrm{e}}$ siècle, l'ange-guide prend de l'importance en raison de l'insistance de la pastorale sur le 
salut personnel. Dès les années 1330, le Pèlerinage de l'âme de Guillaume de Digulleville mettait en valeur le couple formé par l'âme et son ange gardien. A la fin du Xve siècle, l' Ars moriendi met en scène l'ange et le démon autour du lit du moribond. La mort est le moment clé du combat terrestre entre les deux camps ; l'ange est alors celui qui prépare le mourant, qui oriente ses pensées, qui lui permet de déjouer les ultimes ruses du diable. La dévotion au protecteur individuel est d'autant plus forte que la croyance aux pouvoirs immenses du démon est de plus en plus répandue, théorisée et mise en relief par une puissante iconographie. Satan ne semble plus rattaché à sa nature angélique, mais avoir acquis une autonomie telle qu'il apparaît comme le rival de Dieu.

50 La dévotion à l'ange gardien semble avoir entraîné des pratiques illicites, d'autant que l'Église elle-même accordait aux anges des possibilités d'action concrète. L'invocation des puissances célestes à des fins utilitaires et la transmission de connaissances par les anges caractérisent « l'art angélique », susceptible de barrer la route aux menées du démon. La dévotion à l'ange gardien vient s'insérer dans la conscience de l'assimilation eschatologique des hommes aux êtres célestes ; ceux-ci figurant l'état spirituel espéré par ceux-là. Les moines n'ont plus le monopole de "l'angélicité: les laïcs y accèdent désormais par leur participation à la liturgie et par leur vie dévote. De plus, le contenu même de la notion de vie angélique s'élargit: on prête aux anges des activités intellectuelles ou matérielles, si bien que l'imitation des anges ne se réduit plus à celle de leur fonction contemplative et liturgique. Cette évolution est inséparable de l'intérêt de plus en plus marqué pour les anges inférieurs, c'est-à-dire pour les anges actifs, en contact permanent avec l'univers physique et avec les hommes.

51 Mais la relation à l'ange prend dès $l e \mathrm{xv}^{\mathrm{e}}$ siècle une teinte sentimentale qui affadit sérieusement sa signification. Le mouvement dynamique qui unit le ciel et la terre est moins conçu comme une participation graduelle à la lumière divine que comme un va-etvient des anges gardiens, ces conseillers et intercesseurs dont on sollicite le concours. Dans un tel schéma, la hiérarchie angélique s'estompe et les anges supérieurs, séraphins chérubins et trônes, n'ont plus leur place.

Au sujet de l'image de l'ange, les visionnaires $d u \mathrm{Xv}^{\mathrm{e}}$ siècle ne s'écartent guère de leurs prédécesseurs du XIII ${ }^{\mathrm{e}}$ : Françoise Romaine ou Margerie Kempe voient leur ange gardien sous l'aspect d'un enfant, auquel la première donne l'âge de neuf ans, ce qui est une manière nouvelle d'évoquer son appartenance au dernier des choeurs célestes. Il semble que l'on assiste à un processus de rajeunissement de l'être angélique, qui prend la forme d'une «féminisation » ou d'une « infantilisation». D'abord jeune homme, l'ange devient androgyne, adolescent, enfant, et nourrisson. Car le $\mathrm{x}^{\mathrm{e}}$ siècle engendre l'image du putto, ce syncrétisme de l'ange et de l'amour antique, qui devient l'une des représentations les plus courantes de l'être céleste aux XVI et $\mathrm{XVII}^{\mathrm{e}}$ siècles. Le rajeunissement du surnaturel ne serait-il pas à rapprocher de l'idée même de Renaissance ? C'est la mythologie grécolatine qui est sollicitée dans ce processus, plus que le fond biblique, en dépit de l'allusion évangélique aux anges des enfants (Mt 18, 10). L'image syncrétique du Putto vient s'ajouter au phénomène d'accroissement quantitatif de la présence angélique dans les manuscrits, pour appauvrir singulièrement la charge spirituelle de l'être céleste.

Derrière une apparente uniformité doctrinale, pourtant ébranlée par les secousses des hérésies ou des apports étrangers, on perçoit une modification graduelle du système angélologique de la tradition chrétienne occidentale. Cette évolution est caractérisée par un éloignement du soubassement biblique sur lequel s'était développée la dimension théophanique de l'ange. L'imitation du Christ, dans sa nature humaine et souffrante, l'a 
emporté sur une imitation des anges, conçue sur le mode liturgique et contemplatif, puis sur le mode de l'intégration d'états spirituels hiérarchisés et de la participation à la lumière divine. L'anthropologie angélique n'a pas disparu, mais s'est ouverte à de nouvelles formes, répondant aux besoins des laïcs. En revanche, le déclin de l'ange comme forme de manifestation du Christ et comme puissance médiatrice souligne l'effondrement du sens théophanique biblique. A «l'angélicité» de l'homme a progressivement succédé l'humanité de l'ange. Le processus apparaît solidaire de celui qui concerne le Christ. Devenu confident, conseiller et intercesseur, l'ange éprouve les sentiments humains et adopte les attitudes du dévot. Les tendances opposées de l'iconographie de la fin du Moyen Age, assignant à l'être céleste une corporéité aérienne, lumineuse et féminine, ou au contraire la corporéité lourde du Putto, semblent confirmer l'éclatement de la tradition angélologique. La fin de l'hégémonie du modèle monastique, centré sur l'imitation des anges, la diversification des voies spirituelles issue de l'évolution de la société médiévale, la pression des hérésies, les tendances au syncrétisme intellectuel ou artistique expliquent pour une bonne part l'ampleur de la transformation de la figure angélique et la survivance de l'ange gardien, qui reste seul en tête-à-tête avec l'homme au delà du Moyen Age.

\section{Conclusions}

La place, les fonctions, les formes de représentation des êtres célestes «qualifient » le christianisme occidental, c'est-à-dire lui donnent sa tonalité et révèlent ses inflexions et mutations. Il apparait que le devenir de la thématique angélique est étroitement lié à l'évolution de la dévotion et aux tendances «incarnationnelles» du christianisme. L'épanouissement de la thématique angélique est en grande partie lié à la force persistante des modèles vétérotestamentaires et à l'idéal d'une vie contemplative monastique. La polarisation de la piété sur la personne du Christ, de plus en plus vu en tant que Sauveur crucifié, conduit à articuler plus étroitement le rôle des anges au Christ souffrant et modifie le rapport entre les anges et les hommes. L'humanisation des anges est le corollaire de la dévotion à l'humanité du Christ. Le désir de voir Dieu, qui se traduit dans la multiplication des images de la Trinité, s'exprime aussi dans la représentation visionnaire des anges supérieurs. Mais l'étude des systèmes symboliques révèle que la médiation passe par d'autres canaux que l'ange.

La prise de conscience de ce qu'on pourrait appeler «l'explosion démographique » des images d'anges aux XIV et $\mathrm{XV}^{\mathrm{e}}$ siècles m'a poussé à ouvrir une enquête sur cette période En analysant les images, on constate que la rançon de « l'explosion démographique » des anges est l'affaiblissement de la figure angélique dans sa fonction de médiateur et de messager de Dieu. Se détachent le protecteur, individuel ou collectif, et l'escorte lumineuse de Dieu. L'unité du système de représentation éclate sous l'influence de la résurgence de modèles antiques. L'effondrement du sens théophanique de l'ange et sa déconnexion du substrat biblique sont patents. Il me semble que l'on peut parler d'une disparition partielle de l'ange biblique et d'une véritable rupture dans la tradition iconographique. On est tenté de voir dans ce phénomène majeur le signe d'une évacuation de l'ange du christianisme et le prélude à la sécularisation. Car c'est le sens théophanique de l'ange qui s'estompe à la faveur de cette rupture, et la réduction de l'ange au décoratif qui s'impose. Quel sens peut revêtir une figuration de l'ange qui ne respecte plus la structure même de la tradition judéo-chrétienne? On est passé d'un 
christianisme angélophile à un christianisme où la présence angélique est singulièrement appauvrie, menacée et parfois dénaturée. Seul l'ange gardien a passé en force les portes de l'époque moderne. 